\title{
The Impact of Data Visualization on User Interaction
}

\author{
Mefail Tahiri \\ Professor at State University of Tetova \\ mefailt@gmail.com \\ Ejup Rustemi
}

Dr.Sc. at State University of Tetova

ejuprustemi@yahoo.com

\begin{abstract}
Since the prehistoric times, people have painted various figures inside caves and other places they inhabited. In other words, humans have always wanted to visualize things in order to facilitate their explanation of moments and situations to others. Nowadays, computers on the other hand, have established a bridge for users to interact between themselves, and to make things easier, data scientists have designed visual representations of the data with the goal of making communication easier, and in that way achieving faster results. In this paper we will explain the process of data visualization and its importance for user interactivity.
\end{abstract}

Key words: data, data visualization, interaction, user interface.

\section{Introduction}

Data visualization has been around for quite some time. We can trace its roots all the way to the $18^{\text {th }}$ century, although some go a lot further down history. Of course people didn't use computers back then to display their data; they were mainly drawn representation of important data for a given field. So now, this raises the question, what is different today compared to the past. Well, although at first it doesn't seem that there exists some big change in this aspect, if we dwell deep into the issue, we will see that a lot of things are different. (Kirk, A., Data visualization: a successful design process, 2012) With the introduction of computers, especially from the time when they became tools for general use, they became devices for writing, graphic design, gaming, etc. all these fields require different approach to data visualization. 
Take for example the gaming industry. When we play a game, be it on a desktop computer, laptop or a cell phone, we will see various graphs which represent the player's name, score, health, ammunition, and so on. This practically is data visualization. But people don't always play games, they use their computers to store data about their monthly expenses, work, family pictures, videos, etc. Every one of those requires data visualization. In other words, when we visualize data, we actually organize data, which is the most important aspect to make any meaning out of them.

\section{Data, data, data,...}

When the ex-Microsoft CEO Steve Ballmer was at a conference, presenting the company's achievements and future goals, while talking about the importance of developers, he started repeating that particular word, over and over again. We did the same thing for the same purpose with our title here. Data are extremely important in every business imaginable. They represent the indicators by which we establish our path towards success. Of course, data require analysis in order to use them in a particular field. To achieve a given analysis with more success, we must visualize data so they become more accessible and easier to manage.

Today, we are highly connected with data in every step we take. We go to a store where we use our credit card to make a purchase, we go to a school where we need to be registered in the school's database, we enter a house where we can be recorded by surveillance camera, etc., etc. all these things have made us highly dependent on data. (Kirk, A., Data visualization: a successful design process, 2012) This can become scary at times in thinking that everything we do is being recorded somewhere, but that's the truth and we need to accept it and find ways how to use the recorded data so it can benefit us in our everyday life.

\section{A picture is worth a thousand words}

Almost all of us has heard this saying. At times it may seem as an exaggeration, but a lot of times it's very near the truth. In other words, we visualize data so we can have a specific view on what we need. By visualizing we can look at graph and in a few seconds understand what's the point and what needs to be done, without wasting time trying to decipher a vast amount of numbers and letters. 
Until now it all sounds ideal and very catchy, but we should keep in mind that data visualization is not an easy task. It may seem easy if we use Microsoft Excel for example, and store some data which it will immediately present them as a pie graph or something similar. But think about the code behind such a presentation, think about the designers who had to think a lot in order to find the most acceptable way to visualize a specific set of data, because we must understand that different data, requires different ways of visualization.

To have a better understanding of what data visualization actually means, we will show the following example.

\begin{tabular}{rrrrrrrrr|}
\hline $\mathbf{x} \mid$ & $\mathbf{y} \mathbf{1}$ & $\mathbf{x 2}$ & $\mathbf{y 2}$ & $\mathbf{x 3}$ & $\mathbf{y 3}$ & $\mathbf{x 4}$ & $\mathbf{y 4}$ \\
10 & 8.04 & 10 & 9.14 & 10 & 7.46 & 8 & 6.58 \\
8 & 6.95 & 8 & 8.14 & 8 & 6.77 & 8 & 5.76 \\
13 & 7.58 & 13 & 8.74 & 13 & 12.74 & 8 & 7.71 \\
9 & 8.81 & 9 & 8.77 & 9 & 7.11 & 8 & 8.84 \\
11 & 8.33 & 11 & 9.26 & 11 & 7.81 & 8 & 8.47 \\
14 & 9.96 & 14 & 8.1 & 14 & 8.84 & 8 & 7.04 \\
6 & 7.24 & 6 & 6.13 & 6 & 6.08 & 8 & 5.25 \\
4 & 4.26 & 4 & 3.1 & 4 & 5.39 & 19 & 12.5 \\
12 & 10.84 & 12 & 9.13 & 12 & 8.15 & 8 & 5.56 \\
7 & 4.82 & 7 & 7.26 & 7 & 6.42 & 8 & 7.91 \\
5 & 5.68 & 5 & 4.74 & 5 & 5.73 & 8 & 6.89 \\
\hline
\end{tabular}

Fig. 1 Sample of statistical data
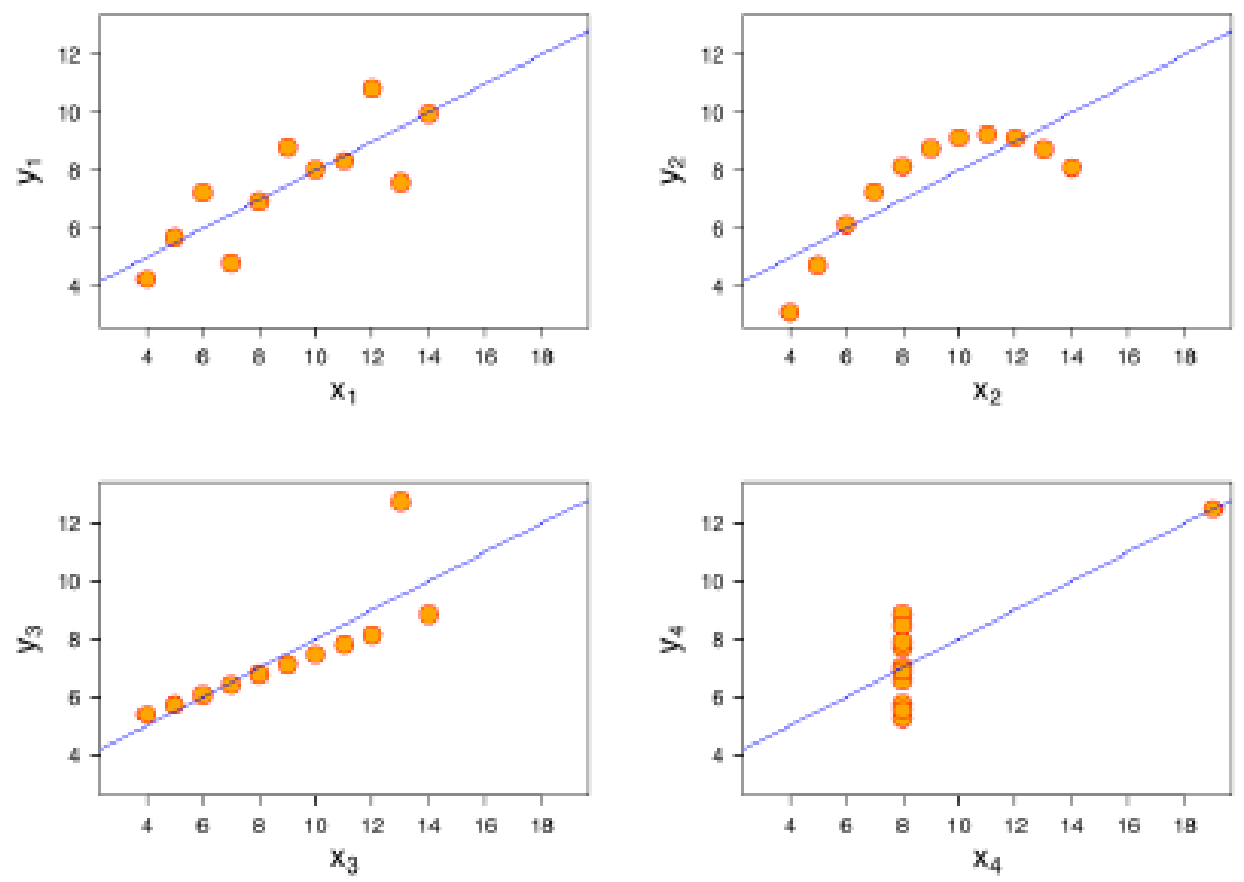
Fig.2 Visualization of the same data

(Kirk, A., Data visualization: a successful design process, 2012)

As we can see from the pictures above, the difference is very big. The first one requires a deep evaluation in order to get any meaning out of it, because it looks like some random numbers are thrown at us and we have to deal with them somehow. Whereas, the second picture is a lot more clear, and we can immediately see the relationship between $\mathrm{X} 1$ and $\mathrm{Y} 1, \mathrm{X} 2$ and $\mathrm{Y} 2$, and so on.

Data visualization is especially important when dealing with the end user. A consumer has no interest in the way you manufacture the shoes you sell, the consumer wants a clean user interface when he will want to purchase them from your website, so he can see a picture of the shoes and the price right beside the picture.

\section{Graphics}

The main tools to visualize our data are called graphics. They may come in different shapes, but the goal is always the same, making it possible for the user to comprehend what he is looking at. If you can remember, we said that data visualization are not new in the age of computer technologies, they have a long history in the human civilization. Emphasizing this, we can say that if we are to mention a graphic representation that has been used since ancient times, it will probably be geographical maps. They are some of the oldest graphical representation known in the human history. Maps as a visualizing tool have been very helpful for all societies.(Myatt, J. G., Johnson, P. W., Making sense of data III, 2011) 
In our age we have digital maps that we can access by using our cell phones. They can have animations, $3 d$ representation, different views such as satellite, road and terrain views.
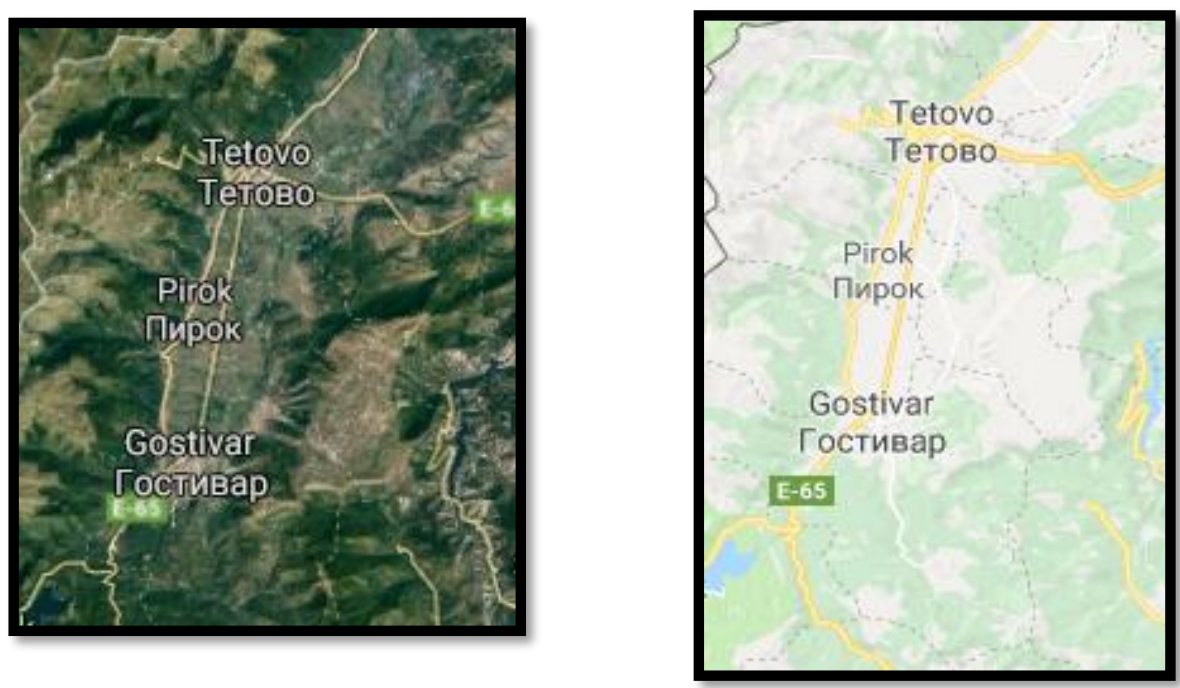

Fig.3 Different visual presentation of geographical data (Google Maps)

To visualize data, a working relationship of a group of people is required. There should be specialists for the given scientific or artistic field, graphic designers, programmers, etc. all of which will cooperate so the end result can be successful.

All in all the process of data visualization can be seen as a two-phase process as suggested by Bertin (fig. 4)

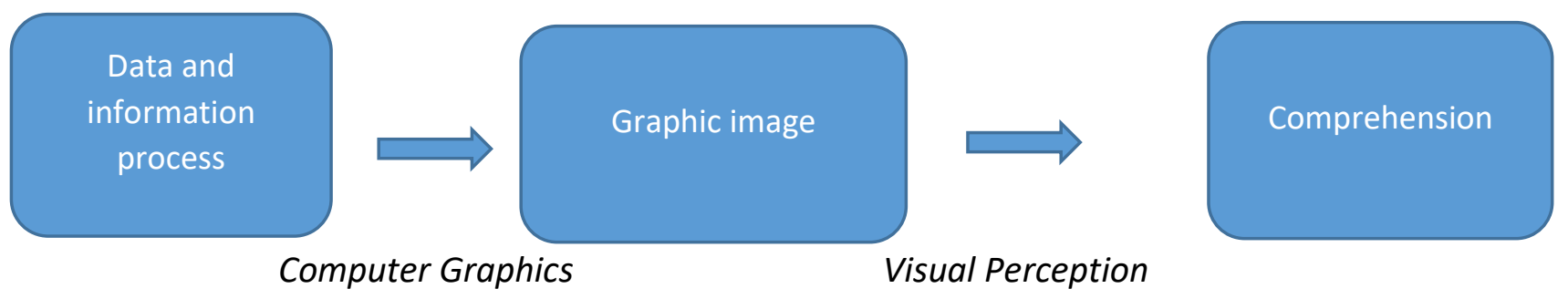

Fig.4 The two-phase process

\section{Where to begin?}

User devices come in various shapes and forms. It may be a laptop computer, a desktop, a cell phone, a smartwatch, or something else entirely. Data designers need to evaluate the platform on which they will develop their application, so they can build quality software and appropriate data visualization. This is very important, because it is not the same when you present data on a 32 " screen 
compared to a 6". Designers must take this issue into consideration if they want to build responsive apps for different devices. Sometimes it will be required to build two or three versions of the same app, based on the usage platform.

Microsoft as one of the IT giants has developed its latest OS while having in mind the facts we mentioned. Windows 10 programmers, are working to make the platform available to all devices, stationary or mobile, so when a developer will make an app, the code will work on both form factors, thus removing the need to recode the same app over and over again. This is very important considering that in today's business landscape, an employee may start working on a desktop PC at work and continue while traveling by bus or train on his cell phone. When the app will be consistent on all platforms, data visualization will benefit from that, and the work will be done in time.

Sometimes, however, data designers, are required to focus on one specific platform where they should visualize the given data.

By the second half of the ' 90 and first half of the ' 00 , the PC market took the world by storm, and that platform was the main focus of developers, data scientists, data designers, etc., but then everything changed. The mobile market exploded. (fig. 5). This required a shift of focus on everything $\mathrm{IT}$, and data visualization in particular. In the beginning, mobile devices didn't have powerful specs, and in order to make them useful for business or entertainment, developers had to scale down the graphical representation of data, to achieve optimum performance.

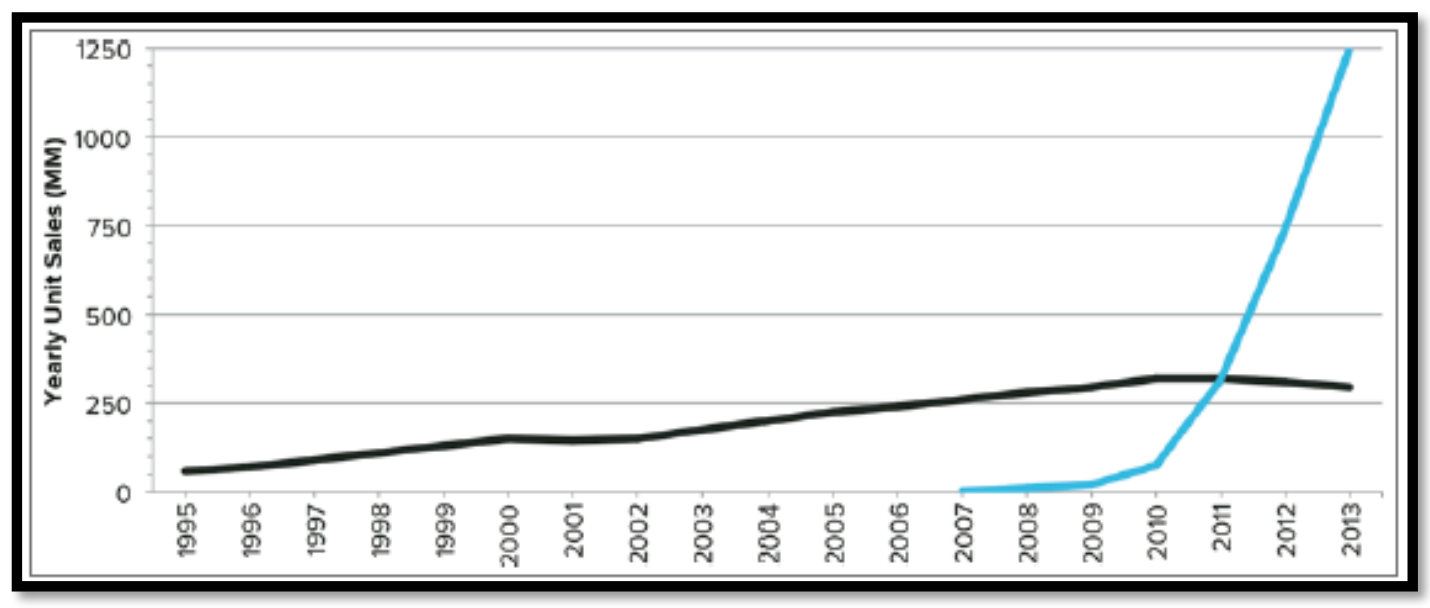

Fig.5 PC and mobile devices sales comparison (black: PC, blue: mobile)

(Hinderman, B., Building Responsive Data Visualization for the Web, 2016) 


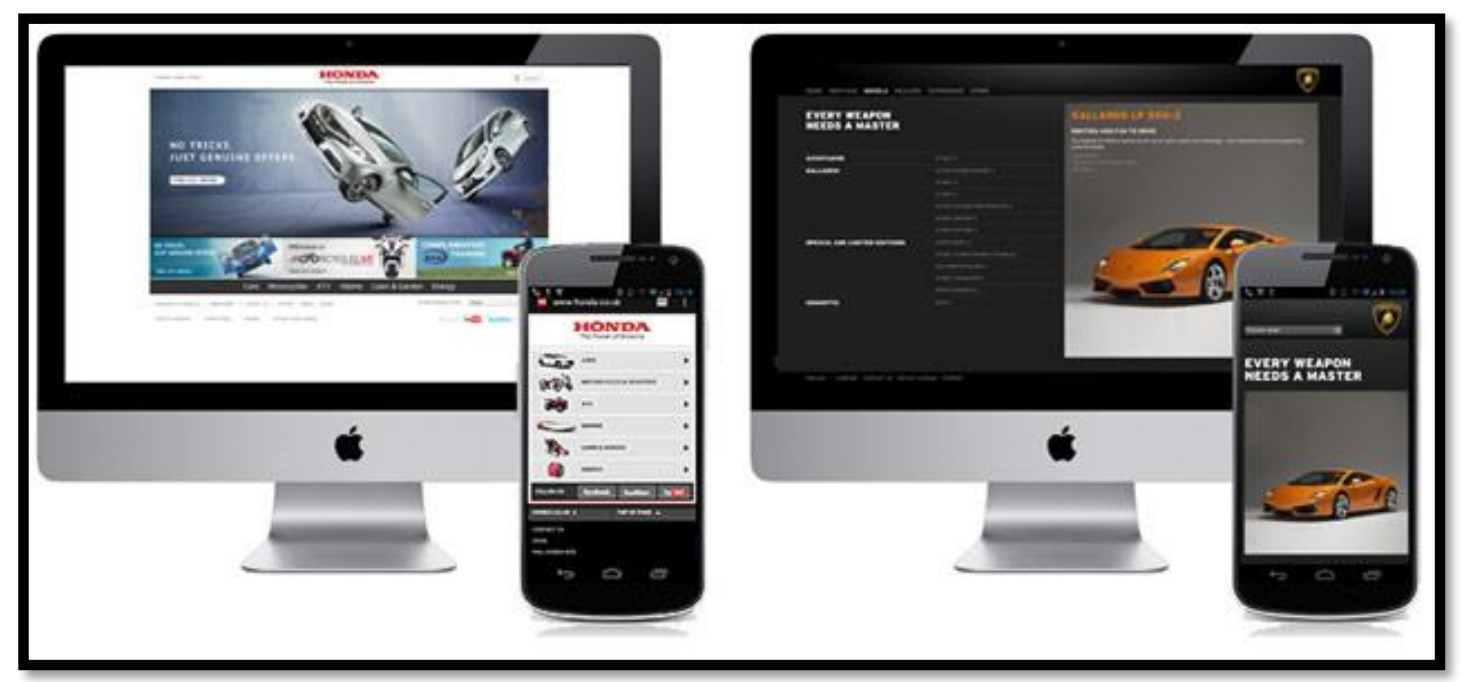

Fig.6 Data visualization comparison (desktop vs. mobile)

(picture taken from: http://www.vividoptimizers.com/2015/08/11/mobile-search-taking-desktopusage/)

The last 3 or 4 years mobile devices have had a huge jump in specs, although still way behind powerful desktop PC-s, still, this means that the gap is getting narrow, especially in relation with visualizing data.

One other thing that data designers should have in mind is to analyze the market, especially the mobile one. There exist a variety of mobile Operating Systems, but only two of them have a vast user community, Google's Android and Apple's iOS platform. At one time the Blackberry's OS showed a promising future, specifically in the enterprise area, but it vanished from the mobile landscape for various reasons. The same can be said about Microsoft's Windows Mobile OS, which also had a short period when it seemed that the platform combined with Microsoft's hugely popular services such as Office, had a great potential, but they failed to gain any important following, so they pulled the plug for the OS.

All these specifics should be known to the data designers, so they can prepare themselves what to expect while visualizing data, if they tend to create a better user interaction. 


\section{The Software}

The IT landscape which encapsulates an enormous software development landscape, has a lot of data visualization tools by which professionals use to design data. Developers of software such as PowerBI, Excel, Google Charts, Visio, IBM OpenDX and other similar visualizing environments, are continuously finding new ways to improve the way we represent our data.

These tools are invaluable because they help data scientist to easily display a given set of data to the world, where users will interact based on what they see.

There is very popular acronym, especially in relation to Game Engines, that goes by WYSWYG, which expands as What You See is What You Get. This basically means that what you are actually seeing is what the developer has intended for, and there is no need to dwell into deep analysis of what a specific number or word means. This is very important when we deal with business data. When we see a pie chart that shows the percentage of male and female students in a particular school, we are actually seeing all that we need, without the need to make any further evaluation or calculation.

Let's consider this example: what if someone gives us the percentage values for various items in a store in the following way: $10 \%, 5 \%, 25 \%, 13 \%, 5 \%, 12 \%, 11 \%, \ldots$ where we need to find the item with the largest quantity. We will have to check and recheck the numbers, to see whether $25 \%$ is the number we are searching for. But what if we are presented with this chart:

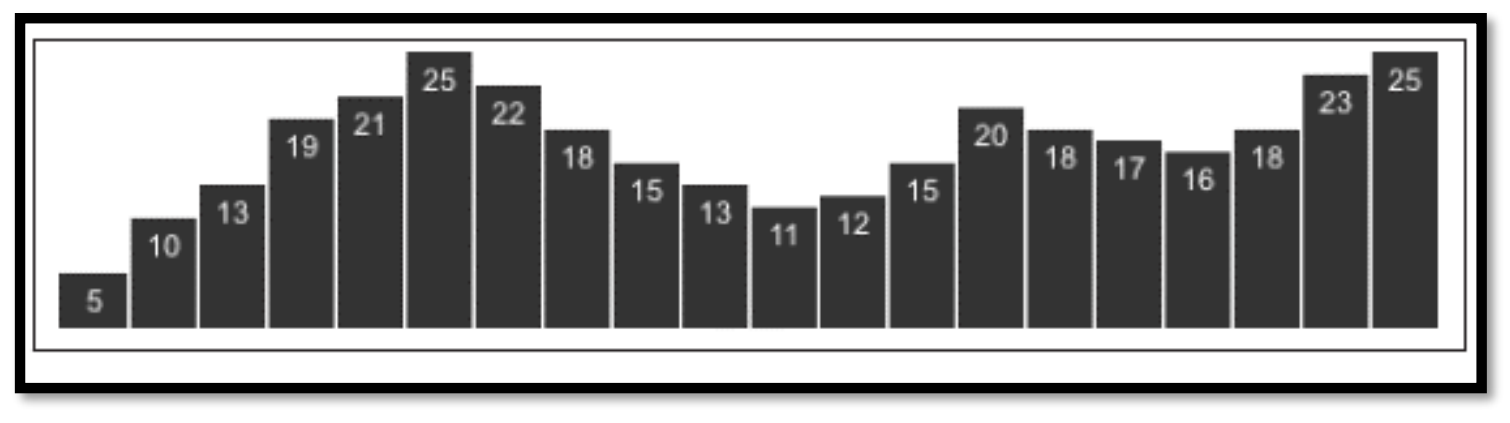

Fig.7 Data visualization

(Murray, S., Interactive Data Visualization, 2010)

With this we will immediately find what we need just by looking at the highest bars. That is what data visualization stands for. 


\section{The Virtual World}

We have seen the Matrix trilogy of movies, where people interact in vast virtual landscapes, fighting viruses that are represented as abominable robots. It seemed only as science fiction at the time when the movies were released, but today we are witnessing a big expansion of virtual reality. In the beginning it was mainly used for the gaming industry where devices such as Oculus Rift gained huge popularity. But in recent years, there is a shift in the usage of virtual devices. Today they are used in many areas ranging from games all the way to architectural visualization. One piece of important technolog needs to be mention here, which goes by the name Mixed Reality, developed by Microsoft. With the introduction of Holo Lens, Microsoft revolutionized the way we use our computers, and in that way data visualization. By using Holo Lens we could interact with the computer in the same way as arranging our room. If by using our PC-s in a conventional way we can store documents in folders and put them on the desktop or any other place in the hard drive, with the new technology we would store them in virtual drawers, or hang our pictures on virtual walls.

This kind of technology is a huge step in the area of data visualization and user interaction. Now an architect can design a house an show it remotely and virtually to the client by walking him around, even though they can be miles and miles apart.

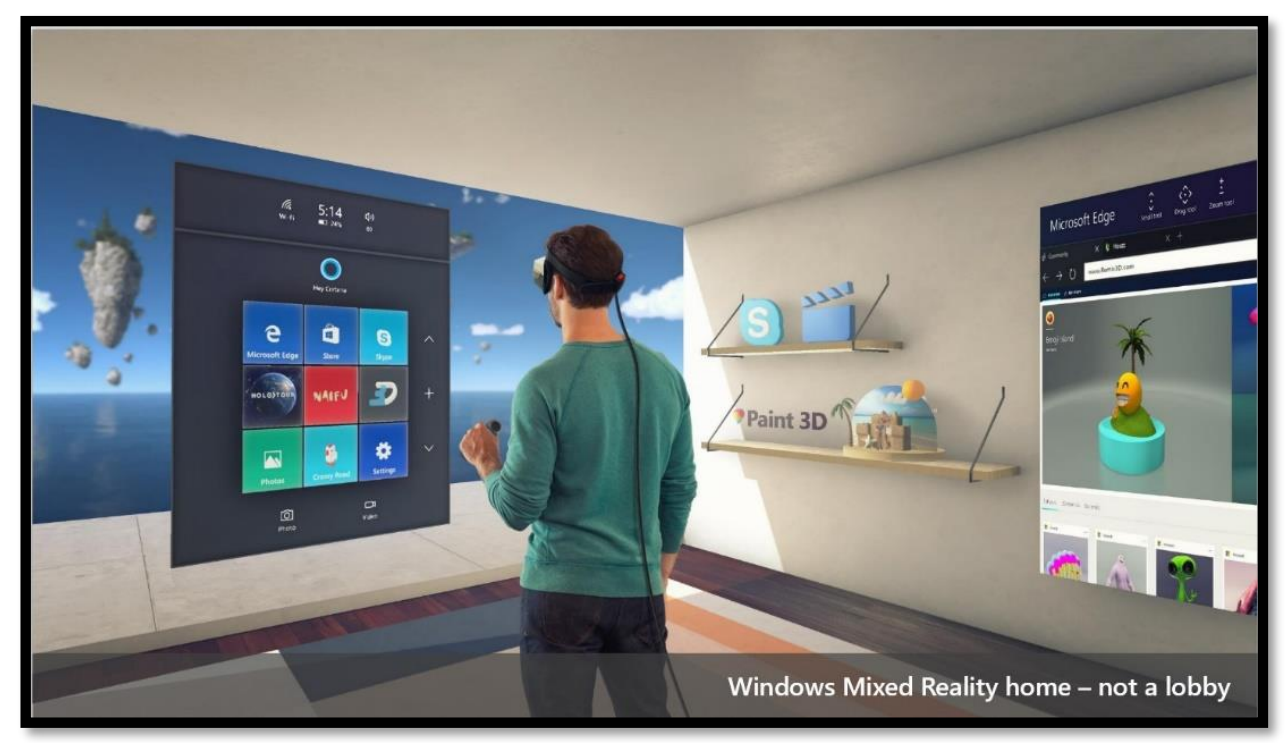

Fig.7 Microsoft's Mixed Reality

(picture taken from: http://www.techradar.com/news/images-show-windows-10s-upcomingmixed-reality-viewer) 


\section{Conclusion}

We believe that we have shown how important data visualization can be. Regardless of the tools we use for visualization, the importance of the technology rests on the fact that it can be applied on every filed of science and art. We have seen that it is a new and an old phenomenon at the same time. No matter how we see it, it seems that we are witnessing the dawn of a true revolution of data design, which will hopefully be of great use to human society, and it will provide us with new methods to improve our lives.

\section{References}

1. Kirk, A., Data visualization: a successful design process, 2012, Packt Publishing, Birmingham

2. Myatt, J. G., Johnson, P. W., Making sense of data III, 2011, Wiley, Hoboken

3. Hinderman, B., Building Responsive Data Visualization for the Web, 2016, Wiley, Indianapolis

4. Murray, S., Interactive Data Visualization, 2010, O'Reilly, Sebastopol

5. http://www.vividoptimizers.com/2015/08/11/mobile-search-taking-desktop-usage/

6. http://www.techradar.com/news/images-show-windows-10s-upcoming-mixed-realityviewer 